\title{
Analysis of Magnetoencephalography Signals from Alzheimer's Disease Patients Using Granger Causality
}

\author{
Celia Juan-Cruz, Carlos Gómez, IEEE Senior Member, Jesús Poza, IEEE Senior Member, \\ Alberto Fernández, and Roberto Hornero, IEEE Senior Member
}

\begin{abstract}
The aim of this study was to analyze resting-state magnetoencephalography (MEG) activity in Alzheimer's disease (AD) by means of Granger Causality (GC), an effective connectivity measure that provides an estimation of the information flow between brain regions. For this task, five minutes of MEG recordings were acquired with a 148-channel whole-head magnetometer from 36 AD patients and 26 healthy controls. Abnormalities in AD connectivity were found in the five typical frequency bands: delta $(\delta, 1-4 \mathrm{~Hz})$, theta $(\theta, 4-8 \mathrm{~Hz})$, alpha $(\alpha, 8-13 \mathrm{~Hz})$, beta $(\beta, 13-30 \mathrm{~Hz})$, and gamma $(\gamma, 30-65$ $\mathrm{Hz})$. Noteworthy increments in delta band and decrements in beta and gamma bands revealed disrupted connections in AD brain activity. Our analyses suggest that GC may be useful to characterize the brain impairment in AD.
\end{abstract}

\section{INTRODUCTION}

Alzheimer's disease (AD), the most common cause of dementia, is a neurodegenerative disorder associated with aging and characterized by progressive memory and cognitive impairment. This disease has an important impact on the social life and development of daily activities of patients and families [1]. Around 46.8 million people worldwide suffer from dementia in 2015 [2]. Among them, it is estimated that around $60 \%$ to $80 \%$ are caused by $\mathrm{AD}$ [3], [4]. Due to the increase in elderly population, $\mathrm{AD}$ is becoming a growing medical concern.

Over the years, several techniques have been used to describe the brain dynamics in AD. Electroencephalography (EEG), magnetoencephalography (MEG), positron emission tomography (PET), and functional magnetic resonance imaging (fMRI) allow to study brain activity in a noninvasive way [5]. In some of the cases, they rely on indirect measures to evaluate the brain activity. For instance, fMRI and PET measure changes in the brain hemodynamics response caused by neural activation, providing an excellent spatial resolution but a poor temporal resolution, which is limited by the rate of oxygen release to neurons (fMRI) or radioactive tracer (PET), and by the blood flow. On the other hand, EEG and MEG provide a direct measure of the

This research was supported in part by 'Ministerio de Economía y Competitividad' and FEDER under project TEC2014-53196-R, 'Consejería de Educación de la Junta de Castilla y León' under project VA059U13, and 'Consejería de Sanidad de la Junta de Castilla y León' under project $\mathrm{BIO} / \mathrm{VA} 08 / 15$

C. Juan-Cruz, C. Gómez, J. Poza and R. Hornero are with the Biomedical Engineering Group (GIB), Signal Theory and Communications Department, University of Valladolid, Paseo de Belén 15, 47011Valladolid, Spain (phone: +34 983423000, ext. 5569; fax: +34 983423667; e-mail: celia.juan@gib.tel.uva.es).

A. Fernández is with the Psychiatry and Medical Psychology Department, Complutense University of Madrid, Avda. Complutense s/n, 28040 - Madrid, Spain electromagnetic brain activity: electric potentials (EEG) or magnetic fields (MEG) [5]. On the contrary to fMRI or PET, temporal resolution is limited by the sampling frequency of the device, achieving resolutions of less than $1 \mathrm{~ms}$. Additionally, magnetic fields are less distorted than electrical activity due to the heterogeneous conductivity of the skull and scalp. Furthermore, MEG provides reference-free recordings and a better spatial resolution than EEG. Consequently, MEG can be considered an appropriate technique for the analysis of brain activity.

Most of the efforts on understanding brain functioning have focused on the evaluation of brain connectivity, as it could be an important tool for the comprehension of the underlying mechanisms associated to a complex disease, such as $\mathrm{AD}$. Connectivity measures are usually differentiated in three categories: anatomical connectivity (structural characteristics of the neural connections in the brain), functional connectivity (dependencies between remote neurophysiological events), and effective or causal connectivity (direction in which that influence is exerted between those events) [6].

In the last decades, numerous studies were conducted to characterize functional connectivity through several mathematical approaches, such as coherence $(\mathrm{COH})$, synchronization likelihood (SL) or phase-lag-index (PLI) [7]-[9], among others. They highlighted abnormalities related to $\mathrm{AD}$ resting-state functional dynamics. Particularly, using EEG, AD evidenced decreased coherences in alpha frequency band and increased in delta band [7]. Other EEG studies revealed a decrease of SL in beta band for AD patients [8]. Using PLI in MEG recordings, Stam et al. [9] found decrements in alpha and beta bands in AD patients compared to healthy controls.

Above-mentioned functional connectivity measurements are helpful tools for the description of brain connectivity. However, they failed to analyze the direction of the interaction between brain regions [10]. In this study, we have examined the spontaneous MEG activity in AD patients using Granger Causality (GC), a popular method able to identify causal connectivity in time-series data. Therefore, the purpose of this study was to characterize connectivity patterns of resting-state MEG in AD.

\section{MATERIALS AND METHOdS}

\section{A. Subjects}

MEG recordings were acquired from 62 subjects. The population was divided into $36 \mathrm{AD}$ patients and 26 controls without past or present neurological disorders. AD patients were recruited from the 'Asociación de Familiares de 
Enfermos de Alzheimer (AFAL)' and the Geriatric Unit of the 'Hospital Clínico Universitario San Carlos' (Madrid, Spain). Diagnoses were made according to the clinical guidelines of the National Institute of Neurological and Communicative Disorders and Stroke and the AD and Related Disorders Association [11]. Cognitive and functional deficits were screened in both groups using the mini-mental state examination (MMSE) and the functional assessment staging (FAST). Differences in the mean age and gender of both populations were not statistically significant $(p=0.19<$ 0.05 , Student's t-test and $p=0.916<0.05$ chi-square test, respectively). Table 1 summarizes the main clinical and socio-demographic data.

\section{B. MEG Recordings}

MEG signals were recorded with a 148-channel wholehead magnetometer (MAGNES 2500 WH, 4D Neuroimaging) placed in a magnetically shielded room at the MEG Center Dr. Pérez-Modrego (Spain). The subjects lay comfortably on a patient bed, in a relaxed state and with their eyes closed, in order to reduce the presence of artifacts in the recordings. Five minutes of MEG data were acquired from each subject at a sampling frequency of $678.17 \mathrm{~Hz}$. A process of down-sampling by a factor of four was carried out to reduce the data length, resulting a sampling rate of 169.55 $\mathrm{Hz}$. Data were digitally filtered using a 1-65 Hz band-pass filter and a $50 \mathrm{~Hz}$ notch filter. Both visual inspection and independent component analysis (ICA) were performed to minimize the presence of artifacts. MEG preprocessed signals were band-pass filtered into the five typical frequency bands: delta $(\delta, 1-4 \mathrm{~Hz})$, theta $(\theta, 4-8 \mathrm{~Hz})$, alpha $(\alpha, 8-13 \mathrm{~Hz})$, beta $(\beta, 13-30 \mathrm{~Hz})$, and gamma $(\gamma, 30-65 \mathrm{~Hz})$. Artifact-free epochs of $5 \mathrm{~s}$ (848 samples) were then selected for further analyses.

\section{Granger Causality (GC)}

GC is a synchrony measure, useful to evaluate the effective connectivity between two signals [12]. As proposed by Wiener in 1956 [13], one signal $x(t)$ could be called causal to other $y(t)$ if it is possible to predict better the second one $y(t)$ by adding past information of the first one $x(t)$ than only with the past information of $y(t)$. This idea was later reformulated using linear autoregressive models for stochastic processes by Granger in 1969 [14]. Specifically, $\mathrm{GC}$ is based in two principles [15]:

1. The cause precedes the effect.

2. Modifications in the cause change the effect.

TABLE I. CLINICAL AND SOCIO-DEMOGRAPHIC DATA OF THE RECRUITED SUBJECTS

\begin{tabular}{|c|c|c|}
\cline { 2 - 3 } \multicolumn{1}{c|}{} & AD patients & Controls \\
\hline Number of subjects & 36 & 26 \\
\hline Age (years) (Mean \pm SD) & $74.06 \pm 6.95$ & $71.77 \pm 6.38$ \\
\hline Gender (F:M) $^{c}$ & $24: 12$ & $17: 9$ \\
\hline FAST $^{\text {a }}$ (Mean \pm SD) & $4.17 \pm 0.45$ & $1.73 \pm 0.45$ \\
\hline MMSE $^{\text {b }}$ (Mean \pm SD) & $18.06 \pm 3.36$ & $28.88 \pm 1.18$ \\
\hline
\end{tabular}

b. FAST: Functional Assessment Staging
Let $X_{1}$ and $X_{2}$ be two systems whose time observations could be represented as the time series $x_{1}(t)$ and $x_{2}(t)$. The univariate autoregressive model (AR) of order $p$ could be formulated as [10]:

$$
\begin{aligned}
& x_{1}(t)=\sum_{k=1}^{p} a_{1}(k) x_{1}(t-k)+u_{1}(t) \\
& x_{2}(t)=\sum_{k=1}^{p} a_{2}(k) x_{2}(t-k)+u_{2}(t)
\end{aligned}
$$

where $a_{i}(k)$ are the model parameters, and $u_{i}(t)$ are the prediction errors for each time. In this case, the prediction of each signal depends only on its own past.

The bivariate autoregressive model of order $p$ is formulated as follows:

$$
\begin{aligned}
& x_{1}(t)=\sum_{k=1}^{p} a_{1,1}(k) x_{1}(t-k)+\sum_{k=1}^{p} a_{1,2}(k) x_{2}(t-k)+w_{1}(t) \\
& x_{2}(t)=\sum_{k=1}^{p} a_{2,2}(k) x_{2}(t-k)+\sum_{k=1}^{p} a_{2,1}(k) x_{1}(t-k)+w_{2}(t)
\end{aligned}
$$

where the prediction errors $w_{i}(t)$ depend on the past of the two signals, and $a_{i, j}(k)$ are the model parameters. According to [14], if the variance of $w_{i}(t)$ is lower than the variance of $u_{i}(t)$ by the inclusion of $a_{i, j}(k)$, then $x_{i}(t)$ is purportedly causing $x_{j}(t)$.

Let $\operatorname{var}\left[u_{i}(t)\right]$ be the variance over time of the prediction errors $u_{i}(t)$ for models with parameters $a_{i, j}(k)=0$, and $\operatorname{var}\left[w_{i}(t)\right]$ the variance of the prediction errors over time for models with $a_{i, j}(k) \neq 0$. Then, GC from $i$ to $j$ is defined as:

$$
G C_{i \rightarrow j}=\ln \frac{\operatorname{var}\left[u_{i}(t)\right]}{\operatorname{var}\left[w_{i}(t)\right]}
$$

Specification of model order $p$ (the total number of past samples taken into account) is required for the calculation of $\mathrm{GC}[16]$. An order $p$ too low gives a sparse representation of the data. On the contrary, high values of $p$ can be problematic for model estimation. In this study, autoregressive model order was selected from the minimum obtained after applying the Akaike and the Bayesian Information Criterion assigning a value of $p=10$ [17].

\section{Statistical Analysis.}

A descriptive analysis was initially performed to explore data distribution (normality and homoscedasticity). Variables did not meet the parametric assumptions. Therefore, MannWhitney $U$-test was used. The significance level was set at $\alpha=0.01$. It is important to note that results were reported only in a descriptive sense, since they were not corrected for multiple comparisons.

Signal processing and statistical analyses were performed using the software packages Matlab (version 7.14 Mathworks, Natick, MA) and the software toolbox Hermes [17].

\section{RESULTS}

GC algorithm was applied to our 148-channel MEG data set. An adjacency matrix was obtained for each subject in each frequency band. Asymmetric matrices of GC median values per group and band are displayed in the first and second column of Figure 1. The third column of this figure 
illustrates statistical results using Mann-Whitney $U$-test. Graph plots summarize statistically significant increases (red) or decreases (blue) in connectivity of $\mathrm{AD}$ patients in comparison with controls for each frequency band.

The analysis showed a widespread significant connectivity increment in delta band for $\mathrm{AD}$ patients compared to controls. On the other hand, GC values decrease in gamma and beta bands for AD patients. A clear asymmetry in the adjacency matrices becomes apparent for theta band with evident decrements in the frontal cortex. Not conclusive results were obtained for alpha band.

\section{DISCUSSION AND CONCLUSION}

The aim of this study was to analyze AD-related alterations in neural information flow. For that purpose, GC was applied to spontaneous MEG recordings from $36 \mathrm{AD}$ patients and 26 control subjects. Our results showed that AD is accompanied by an increase of the causal connectivity in delta band, as well as a decrease in high-frequency bands (i.e., beta and gamma bands). $\delta$

AD patients

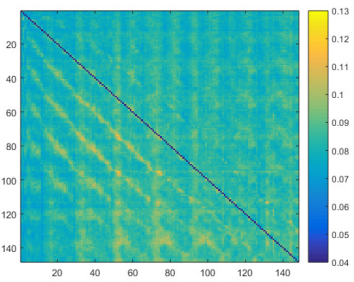

$\Theta$

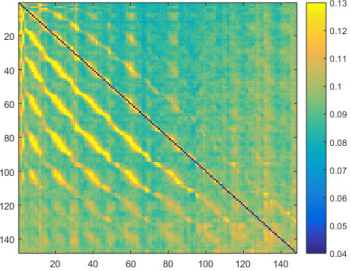

$\alpha$

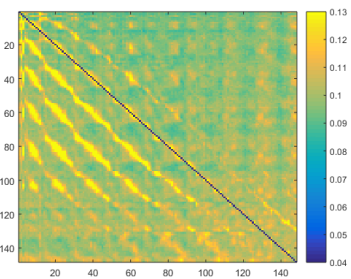

$\beta$

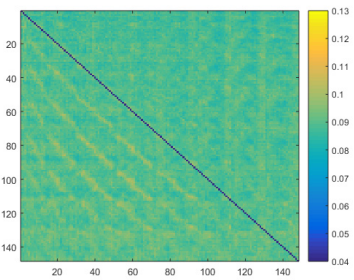

Y

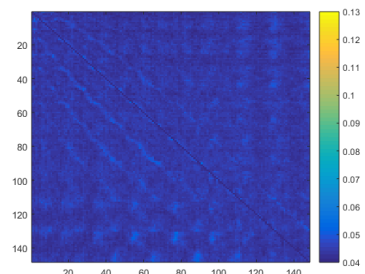

Controls
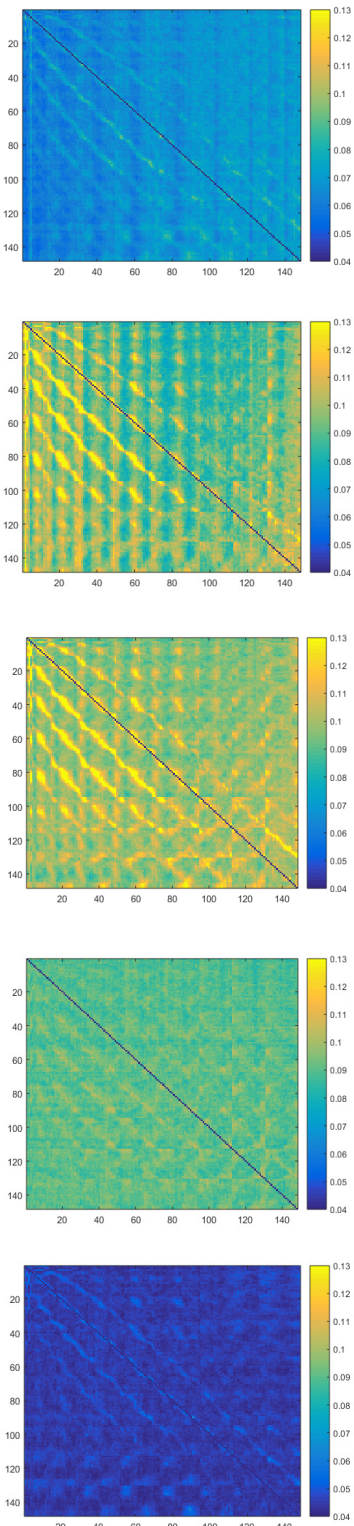

Connectivity Graphs
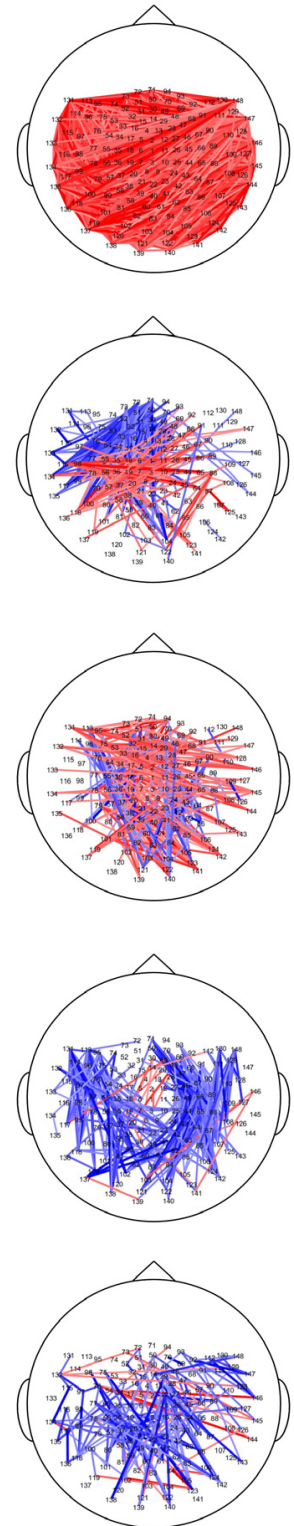

Figure 1. Results obtained after applying GC to MEG recordings. First and second column correspond to the median GC results for AD patients and controls, respectively. Third column illustrates statistically significant increases (red) or decreases (blue) in connectivity values of AD patients respect to controls for each frequency band. For simplicity, connectivity graphs do not show the direction of each connection. 
Our findings are consistent with previous works that evidenced increments in AD activity in delta band. Specifically, in [7] and [18] the increment in delta coherence and Global Field Synchronization (GFS) was attributed to the cholinergic deafferentation, characteristic in AD.

Theta band is clinically associated with working memory processes, which are usually reduced in $\mathrm{AD}$ [8]. A decrease in the fronto-cortical region is found in our results, which is in concordance with [19]. The asymmetry of the adjacency matrices in this band suggests the presence of a strong direction of the information flow, which is less pronounced in AD subjects.

Our results in alpha band are less forthright. Although, several studies reported decrements in alpha band in EEG [7] and MEG [9], our findings are inconclusive, showing increases and decreases in connectivity in similar brain regions. Nevertheless, the direction of the coupling in this band is noticeable in the adjacency matrices for both groups. This direction appears to be stronger for control subjects.

In the case of beta band, several studies reported weak couplings in AD subjects compared with healthy controls [9], [18], [20]. A loss in gamma band synchronization was also reported for the first time in [21]. These results are in accordance with our GC results in beta and gamma bands, and reinforce the hypothesis of disconnection syndrome in $\mathrm{AD}$.

Classically, connectivity decrements were attributed to neuronal loss and neocortical disconnection [19]. However, this could not explain the existing dissimilarities among bands, as was pointed by [8]. In the aforementioned study, the differences in connectivity patterns were attributed to loss of cortical acetylcholine synthesis, which is involved in synaptic function.

Some limitations in this study should be taken into account. Although GC results present asymmetric adjacency matrices indicating causal connectivity, a broad study should be done to completely characterize the direction of the couplings. It should be also noted that GC analysis was performed by means of bivariate autoregressive models and indirect connections will be present. In addition, it should be further explored whether the abnormal GC patterns are ADspecific and confirm the GC results with alternative effective connectivity measures such as Partial Directed Coherence (PDC) or direct Directed Transfer Function (dDTF).

In summary, this research shows that $\mathrm{AD}$ elicits an abnormal frequency-dependent pattern of effective connectivity. In our study, high-frequency couplings seem to be disrupted. This agree with the hypothesis of a disconnection syndrome in $\mathrm{AD}$, since these bands are related to cognitive processes, which commonly are impaired in this disease [18]. Our findings suggest that GC can be useful to discriminate $\mathrm{AD}$ patients, as it evaluates both the connection between groups of neurons, and the direction in which that coupling is exerted. That information can provide further insights into neural mechanisms underlying AD.

\section{REFERENCES}

[1] H. Brodaty and M. Donkin, "Family caregivers of people with dementia," Dialogues Clin. Neurosci., vol. 11, no. 2, pp. 217-228,
2009.

[2] M. Prince, A. Wimo, M. Guerchet, A. Gemma-Claire, Y.-T. Wu, and M. Prina, "World Alzheimer Report 2015: The Global Impact of Dementia - An analysis of prevalence, incidence, cost and trends," Alzheimer's Dis. Int., p. 84, 2015.

[3] Alzheimer's Association, "2015 Alzheimer's disease facts and figures.," Alzheimer's Dement. J. Alzheimer's Assoc., vol. 11, no. 3, pp. 332-84, 2015.

[4] S. Seshadri, A. Beiser, J. Selhub, P. F. Jacques, I. H. Rosenberg, R. B. D'Agostino, P. W. F. Wilson, and P. A. Wolf, "Plasma Homocysteine As a Risk Factor for Dementia and Alzheimer' S Disease," N. Engl. J. Med., vol. 346, no. 7, pp. 476-483, 2002.

[5] E. L. Hall, S. E. Robson, P. G. Morris, and M. J. Brookes, "The relationship between MEG and fMRI,” Neuroimage, vol. 102, no. P1, pp. 80-91, 2014.

[6] K. J. Friston, "Functional and effective connectivity: a review," Brain Connect., vol. 1, no. 1, pp. 13-36, 2011.

[7] T. Locatelli, M. Cursi, D. Liberati, M. Franceschi, and G. Comi, "EEG coherence in Alzheimer's disease," Electroencephalogr. Clin. Neurophysiol., vol. 106, pp. 229-237, 1998.

[8] C. J. Stam, V. D. M. Y, P. Yal, and S. P. Eeg, "EEG synchronization in mild cognitive impairment and Alzheimer' s disease," Acta Neurol. Scand., vol. 108, no. 13, pp. 90-96, 2003.

[9] C. J. Stam, W. De Haan, A. Daffertshofer, B. F. Jones, I. Manshanden, A. M. Van Cappellen Van Walsum, T. Montez, J. P. A. Verbunt, J. C. De Munck, B. W. Van Dijk, H. W. Berendse, and P. Scheltens, "Graph theoretical analysis of magnetoencephalographic functional connectivity in Alzheimer's disease," Brain, vol. 132, no. 1, pp. 213 $224,2009$.

[10] B. Gourévitch, R. Le Bouquin-Jeannès, and G. Faucon, "Linear and nonlinear causality between signals: Methods, examples and neurophysiological applications," Biol. Cybern., vol. 95, no. 4, pp. 349-369, 2006

[11] G. Mckhann, D. Drachman, M. Folstein, R. Katzman, D. Price, and E. M. Stadlan, "Clinical diagnosis of Alzheimer's disease: report of NINCDS-ADRDA work group under the auspices of department of health and human services task force on Alzheimer's disease," Neurology, vol. 34, no. 7, pp. 939-944, 1984.

[12] J. Dauwels, F. Vialatte, T. Musha, and A. Cichocki, "A comparative study of synchrony measures for the early diagnosis of Alzheimer's disease based on EEG," Neuroimage, vol. 49, no. 1, pp. 668-693, 2010.

[13] N. Wiener, The theory of prediction. McGraw-Hill New York, 1956.

[14] C. W. J. Granger, "Investigating Causal Relations by Econometric Models and Cross-spectral Methods," Econometrica, vol. 37, no. 3, pp. 424-438, 1969.

[15] M. Eichler, "Causal inference in time series analysis," in Causality: statistical perspectives and applications., 2012, pp. 327-354.

[16] S. L. Bressler and A. K. Seth, "Wiener-Granger Causality: A well established methodology," Neuroimage, vol. 58, no. 2, pp. 323-329, 2011.

[17] G. Niso, R. Bruña, E. Pereda, R. Gutiérrez, R. Bajo, F. Maestú, and F. Del-Pozo, "HERMES: Towards an integrated toolbox to characterize functional and effective brain connectivity," Neuroinformatics, vol. 11, no. 4, pp. 405-434, 2013

[18] T. Koenig, L. Prichep, T. Dierks, D. Hubl, L. O. Wahlund, E. R. John and V. Jelic, "Decreased EEG synchronization in Alzheimer's disease and mild cognitive impairment," Neurobiol. Aging, vol. 26, no. 2, pp. 165-171, Mar. 2005.

[19] C. Besthorn, H. Förstl, C. Geiger-Kabisch, H. Sattel, T. Gasser, and U. Schreiter-Gasser, "EEG coherence in Alzheimer disease," Electroencephalogr. Clin. Neurophysiol., vol. 90, no. 3, pp. 242-245, 1994.

[20] J. F. Alonso, J. Poza, M. Á. Mañanas, S. Romero, A. Fernández, and R. Hornero, "MEG connectivity analysis in patients with Alzheimer's disease using cross mutual information and spectral coherence," Ann. Biomed. Eng., vol. 39, no. 1, pp. 524-536, 2011.

[21] C. J. Stam, A. M. V van Walsum, Y. A. L. Pijnenburg, H. W. Berendse, J. C. de Munck, P. Scheltens, and B. W. van Dijk, "Generalized synchronization of MEG recordings in Alzheimer's disease: Evidence for involvement of the gamma band," J. Clin. Neurophysiol., vol. 19, no. 6, pp. 562-574, 2002. 